\title{
Developing Global Insight into RNA Regulation
}

\author{
R.B. DARNELL \\ Howard Hughes Medical Institute, Laboratory of Molecular Neuro-Oncology, \\ The Rockefeller University, New York, New York 10021
}

\begin{abstract}
Systematic dissection of the activity of RNA-binding proteins (RBPs) has begun to yield global insight into how they work. The paradigm we have used has been the study of Nova, a neuron-specific RBP targeted in an autoimmune neurologic disorder associated with cancer. We have developed a combination of biochemical, genetic, and bioinformatic methods to generate a global understanding of Nova's role as a splicing regulator. Genome-wide identification and validation of Nova target RNAs have yielded unexpected insights into the protein's mechanism of action, its role in neurobiology, and the unique roles RBPs have in the biology of the neuronal synapse. These studies provide us with a paradigm for understanding the role of RBPs in neurons and in disease and, more generally, with the hope that it will be feasible to develop a comprehensive understanding of posttranscriptional regulation.
\end{abstract}

\section{RBPS IN NEURONS AND NEUROLOGIC DISEASE}

RNA has greater complexity than DNA. RNA folds into complex shapes, utilizing both sequence and structure, allowing it to harbor both information content and enzymatic activity. From a single DNA template, RNA can offer combinatorial complexity (via alternative splicing of exons or RNA editing), as well as the ability to regulate protein expression in space and time (Darnell 2002). Given the need for complexity in the nervous system, where there is an intuitive mismatch between the approximately $2 \times 10^{4}$ primary transcripts encoded by DNA and the approximately $10^{11}$ neurons, each of which may harbor about $10^{7}$ synaptic connections needing regulation, it is natural to wonder whether RNA regulation might contribute to neuronal complexity.

The first clue that neurons might have a unique system for regulating RNA metabolism came from studies of the intersection between cancer cells and neurons. Ron Evans and colleagues' interest in the study of calcitonin, a hormone expressed in the thyroid gland (and, although not as well appreciated, also in the brain) led them to study a medullary carcinoma of the thyroid tumor cell line as a model for comparative gene expression studies. These authors found that a unique transcript of the calcitonin gene was expressed in the tumor cell line and, in further studies, that this was an alternatively spliced isoform normally expressed in the brain. This isoform turned out to encode a completely different protein - calicitoningene-related peptide-from the primary "calicitonin" pre-mRNA transcript (Rosenfeld et al. 1983). These studies established two interesting points. First, there was the possibility that the brain may have its own special system for regulating RNA expression, able to generate unique RNA and hence protein isoforms from a single primary transcript. Second, analyzing the dysregulation of gene expression in tumor cells might paradoxically yield insight into neuron-specific gene expression.

The second insight was extended in a more systematic fashion through studies of a group of neurologic disorders termed the paraneoplastic neurologic disorders (PNDs) (Darnell and Posner 2003b, 2006). Interestingly, these disorders also manifest at the intersection of tumor and neurobiology. PND patients present with specific neurodegenerative syndromes, which can vary widely between patients, and include memory loss, blindness, cerebellar dysfunction, and motor or sensory disorders. For each set of neurologic symptoms, there are characteristic tumors present in these patients, although typically they have not yet presented clinically at the time neurologic illness sets in. These tumors have not invaded the nervous system and are typically limited in their extent of spread. A model for the pathogenesis of the disorders is based on the findings of Jerome Posner and colleagues, who in the early 1980s first found evidence of an immunologic link between cancer and neurologic syndrome (reviewed in Darnell 1996). The model proposes that tumors present in PND patients initiate the syndrome when they express proteins that are normally restricted in their expression to the nervous system. Because there is a blood-brain barrier, the immune system is able to mount what turns out to be an effective immune response to neuronal antigens expressed in peripheral tumors. This accounts for the occult nature of the tumors in these patients and the limited stage of their disorders. In fact, PNDs provide what is perhaps the best model for naturally occurring tumor immunity in humans (Darnell and Posner 2003a). Patients do not present to clinicians until some poorly understood event allows this immune response to break the immunologic blood-brain barrier and then attack those neurons that were normally expressing the neuronal antigens co-opted by the tumor. Although the details of disease pathogenesis remain under investigation (Albert and Darnell 2004), our laboratory established methods to use the high-titer antibodies in PND patients to screen expression cDNA libraries and to identify the genes encoding a number of target PND antigens (Newman et al. 1995; Darnell 1996). To date, more than a dozen such genes have been identified (Darnell and Posner 2006). 
One set of PND antigens that tumor cells consistently express are neuron-specific RBPs. Two families of such proteins were discovered by using PND antisera to screen cDNA libraries - the Nova proteins (Buckanovich et al. 1993), targeted in patients harboring lung or gynecologic cancers and manifesting by neurologic symptoms of excess motor movements (paraneoplastic opsoclonusmyoclonus ataxia [POMA]), and the $\mathrm{Hu}$ proteins (Szabo et al. 1991). Although the functions of the Hu proteins in the brain are still incompletely understood (Musunuru and Darnell 2001), we have been able to establish that Nova regulates neuron-specific alternative splicing in an interesting subset of pre-mRNAs (Ule and Darnell 2006). Studies of the Nova proteins have established a crude template for attempting to understand RBP function on a genome-wide scale. We review here this template, which includes three main components-biochemical, genetic, and bioinformatic - that together provide a means to identify the in vivo RNAs bound by Nova and the functional understanding that results from this template.

\section{BIOCHEMICAL UNDERPINNINGS TO UNDERSTANDING RBP FUNCTION}

An essential foundation in approaching RBP function is a detailed understanding of the nature of the proteinRNA interaction. As a first step, this can be approached in an idealized setting in vitro, with the goal of yielding as detailed an understanding as possible, preferably by X-ray crystallography, of the means by which an RBP recognizes its RNA substrate.

For Nova, which we found to be an RBP harboring three KH-type RNA-binding domains (Buckanovich et al. 1996), we approached this problem by undertaking in vitro RNA selection experiments, using protocols established by Jack Szostak and colleagues (Green et al. 1991; Szostak and Ellington 1993). Two different sets of experiments were done. First, an idealized Nova target RNA was identified using long random RNA libraries and full-length recombinant Nova protein. This led to the identification of a stem-loop RNA harboring a core 4-nucleotide repeat sequence-(UCAU) $)_{3}$ - present in the loop (Fig. 1) (Buckanovich and Darnell 1997; Yang et al. 1998). Mutagenesis studies identified the CA dinucleotide as a critical invariant component of binding, with some flexibility allowed in the flanking $U$ nucleotides and in the stem. Second, these studies were complemented by collaboration with the crystallography laboratory of Stephen Burley, who proposed solving the crystal structure of a single $\mathrm{KH}$

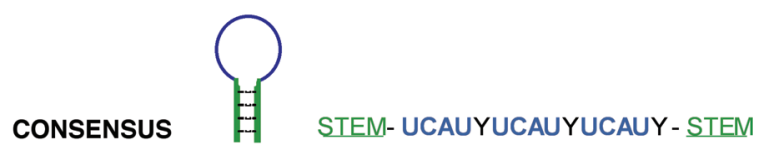

Figure 1. Schematic of the consensus RNA selected with recombinant Nova fusion protein. Nova recognizes RNAs harboring a core loop element (blue) consisting of YCAY repeats (as determined by mutagenesis studies), presented in the context of an inverted repeat forming a stem element. (Adapted, with permission, from Buckanovich and Darnell 1997. [C ASM].)

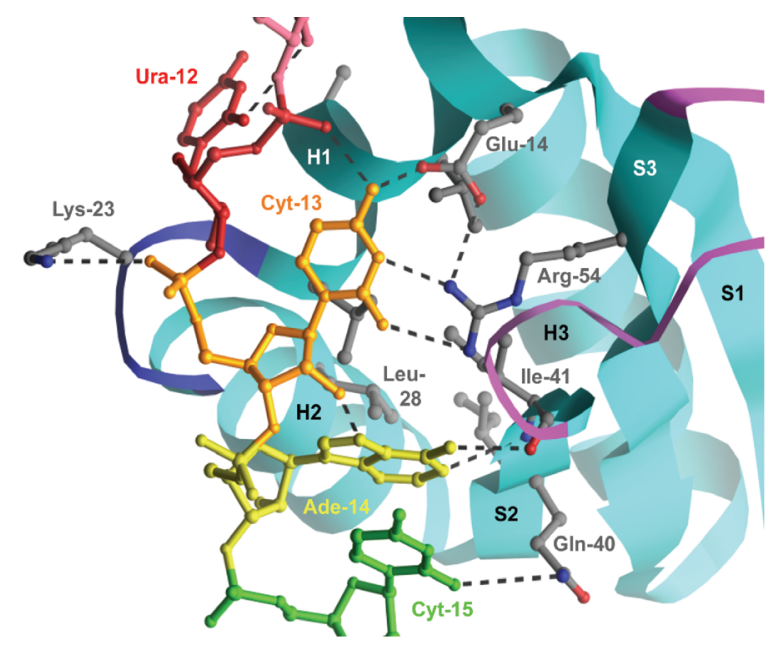

Figure 2. X-ray crystallographic structure of the Nova $\mathrm{KH} 3$ domain bound to RNA. The KH3 domain (turquoise ribbon) folds such that several side-chain amino acids (e.g., Glu-14 and Arg-54) are precisely positioned to provide appropriate hydrogen bond donor/acceptor groups to RNA (colored stick figure). The most precise coordination is for Cyt-13 and Ade-14 (the "CA" core of the YCAY motif), such that the hydrogen bonds are precisely those that would normally be supplied by a second-strand nucleic acid (e.g., "GT"), in this way exactly specifying the sequence. The surrounding nucleotides (Ura-12 and Cyt-15) are also restricted, but less precisely, to pyrimidine residues. (Adapted, with permission, from Lewis et al. 2000 [C Elsevier].)

domain bound to RNA. Two experiments were key to solving this structure. First, Burley's lab undertook a detailed analysis of the structure of Nova by limited proteolysis, delimiting a core protease-resistant region surrounding the $\mathrm{KH}$ domains that would prove to successfully form crystals. Repeating these studies in the presence of RNAs also provided a crucial result, identifying a carboxy-terminal extension on the $\mathrm{KH}$ domain that was protease-resistant only when Nova was bound to RNA, suggesting a role in RNA binding (Lewis et al. 1999). Second, we repeated RNA selection experiments to optimize an RNA target for crystallography with the defined, crystallizable, Nova KH3 domain (Jensen et al. 2000a). Putting these two experiments together yielded a high-resolution X-ray structure of the Nova KH3 domain bound to RNA (Fig. 2) (Lewis et al. 2000). This structure confirmed and extended our biochemistry data, demonstrating that the Nova KH domain folds to position side-chain amino acids to precisely contribute hydrogen bond donor/acceptors in the same manner a second-strand nucleic acid would, to exactly specify the CA core dinucleotide. The KH domain also delimited the flanking residues to be pyrimidines ("Y"), whereas the stem was not bound directly and appeared to function largely to keep the YCAY core element in the loop unbound to other RNA residues and thereby free for protein interaction.

\section{GENETIC SYSTEMS AND RBP FUNCTION}

The recognition of YCAY elements as the core component specified by the Nova $\mathrm{KH}$ domain encouraged us to search for brain transcripts that might encode 
repeats of these elements and hence by Nova targets. Our first very crude approach to this problem yielded one fortuitous hit. Using Microsoft Word as a search tool, we examined by hand intronic and exonic sequences present in a database of 350 neuronal transcripts that had been established at CSHL by Stamm and Helfman (Stamm et al. 1994). We identified the YCAY cluster in this database within an intronic sequence upstream of an alternatively spliced exon (E3A) of the inhibitory glycine receptor $\alpha 2$ (GlyR $\alpha 2)$. Generation of a minigene encoding this element and the surrounding exons demonstrated that in transfected tissue culture cells, Nova was able to mediate an increase in inclusion of E3A. This led to the hypothesis that Nova might regulate alternative splicing of this, and other transcripts, in neurons.

We then crossed a biologic threshold, committing to testing this hypothesis in vivo by generating Nova null mice. These studies proved to be crucial, as they not only gave a critical test of our biochemical data, but also allowed us to develop a robust genetic template for use in further biochemical studies. Assessing GlyR $\alpha 2$ splicing in Noval KO mice, we found a consistent twofold decrease in E3A utilization (Jensen et al. 2000b), consistent with the increase mediated by excess Nova in tissue culture cells (Buckanovich and Darnell 1997).

To assay the specificity of Nova's action, we analyzed splicing of a small set of alternative exons in Nova KO mouse brain. We found that other alternatively spliced brain transcripts were unaffected by the presence or absence of Nova, indicating the specificity of Nova's action, but with one exception, the $\gamma 2 \mathrm{~L}$ exon of the $\mathrm{GABA}_{\mathrm{A}}$ transcript (Jensen et al. 2000b). Given that Nova was targeted in a PND in which patients had an excess motor activity - interpreted by neurologists to be a defect in inhibitory motor control - the finding that two of two Nova-regulated transcripts encoded inhibitory neurotransmitter receptors was tantalizing but inconclusive, given the relatively arbitrary nature in which these transcripts had been identified. This frustration helped fuel a push to develop new methods for genome-wide identification of Nova targets, in order to test the hypothesis that Nova might regulate a restricted set of mRNAs whose functions relate to the neurology of the Nova PND syndrome. Throughout these studies, however, we followed the paradigm established above: Candidate Nova targets, identified by any method, would need to be validated in vivo in a biologically relevant genetic system (Nova KO vs. WT brain) and supported, where feasible, by biochemical studies.

\section{BIOINFORMATICS, GENETICS, AND BIOCHEMISTRY: THE HOLISTIC APPROACH TO RBP FUNCTION}

An additional advantage of identifying the $\mathrm{GABA}_{\mathrm{A}}$ transcript as a Nova target was that it was done independently of a search for specific (e.g., YCAY) binding sequences, thereby allowing us to search blindly for a necessary and sufficient element in the $\mathrm{GABA}_{\mathrm{A}}$ transcript able to mediate the action of Nova on $\gamma 2 \mathrm{~L}$ exon inclusion. These studies revealed a core 24-nucleotide element able to confer Nova-dependent splicing on a heterologous transcript, and sequencing of this element revealed that it was full of YCAY elements (Dredge and Darnell 2003). Biochemical studies of this and one additional Nova-regulated exon (an autoregulated exon in the Nova1 transcript itself) (Dredge et al. 2005) suggested that a core cluster of three YCAY elements was critical in Nova-mediated regulation of splicing.

Taken together, these observations set the groundwork for undertaking a bioinformatic screen for Nova target transcripts (Ule et al. 2006). We developed an algorithm to score transcripts as potential Nova targets on the basis of their YCAY clusters. We used this algorithm to search a set of approximately 50 known Nova targets (including those identified by new methods described below). These studies identified YCAY clusters in Nova targets that had been identified and validated independently of their sequence composition, supporting the importance of this RNA motif in mediating Nova action. Importantly, the YCAY cluster scoring algorithm was robust enough to predict 30 RNA targets based on the presence of YCAY clusters, and these all proved to be bona fide targets when tested in Nova KO versus WT brains (Ule et al. 2006). This helped validate the algorithm itself, as well as lending further support to the significance of YCAY as a biologically relevant Nova-binding motif.

\section{NOVA RNA MAP}

These bioinformatic studies yielded an unexpected finding: The position of the YCAY cluster systematically correlated with the action of Nova on alternative exon inclusion or exclusion (Ule et al. 2006). Clusters immediately upstream or within alternative exons predicted an action of Nova to inhibit exon inclusion, whereas clusters downstream from alternative exons predicted an action to enhance exon inclusion. Thus, a very tightly defined RNA-binding map was generated, in which both the sequence and position of Nova-binding elements determined function.

Such studies clearly have mechanistic implications, suggesting that the position of Nova binding to premRNA precisely relates to its effect on the splicing machinery. Thus, it was logical at this point to undertake definitive mechanistic studies to evaluate this issue. In vitro splicing assays were established, using two sets of model pre-mRNAs, those in which Nova either enhanced or inhibited exon inclusion. In both systems, we were able to demonstrate that purified Nova was able to either enhance or inhibit exon inclusion in a manner consistent with this RNA map, dependent on both the sequence and position of YCAY elements. In parallel, quantification of splicing intermediates in Nova $\mathrm{KO}$ brain demonstrated a direct and asymmetric action of Nova on introns harboring or proximate to YCAY-binding elements, suggesting a mechanistic model distinct from a role in exon definition, but rather one in which Nova might act locally on spliceosome assembly. Consistent with this idea, in vitro splicing assays were able to demonstrate actions of Nova on the formation of the 
basal machinery of the spliceosome; for example, Nova inhibition of splicing by binding at the $3^{\prime}$ end of an alternative exon led to inhibition of U1 small nuclear ribonucleoprotein (snRNP) binding and hence inefficient exon inclusion (Ule et al. 2006).

\section{HOLISTIC PIONEERING: NEW METHODS TO UNDERSTAND RBP FUNCTION}

An essential ingredient in developing a global understanding of RBP function is defining a robust list of biologically validated RNA targets. Several approaches have been considered by different laboratories (Blencowe 2005, 2006), although some methods have had difficulty in generating consistent results. Among these, perhaps the most widely used has been one in which RBPs of interest are immunoprecipitated, and RNA identified in the precipitates by reverse transcriptase-polymerase chain reaction (RT-PCR). Problems with this approach include precipitation (typically done under low-to-moderate stringency) of additional associated RBPs along with their RNA targets, signal:noise problems, and reassociation of RBPs with new RNA targets during immunoprecipitation (Mili and Steitz 2004).

The ability to validate Nova targets, by combining biochemical, genetic (Nova KO), and bioinformatic (YCAY cluster) analyses, gave us a base from which to try to develop new methods to overcome problems in identification of valid in vivo protein-RNA interactions. We undertook two new approaches to identifying Nova targets, one biochemically based, and a second microarray/ bioinformatics based.

The first of these methods, termed CLIP (for crosslink-immunoprecipitation) (Ule et al. 2003, 2005a), takes advantage of a long-standing biochemists' trick, which is the finding that UV-B irradiation is able to induce covalent complexes between protein-nucleic acid (but not protein-protein) interactions, when contact distances are within about $1 \AA$. By applying UV-irradiation to acutely dissected mouse brains, we were able to covalently cross-link Nova-RNA complexes in situ. Once formed, these complexes are extremely stable (samples can be frozen and stored for future experiments), and an RBP could be rigorously purified (to homogeneity if desired). For Nova, our purification was severalfold: immunoprecipitation with a high-titer specific antibody under very stringent conditions, boiling complexes in SDS-sample buffer, running complexes on SDS-polyacrylamide gel electrophoresis (SDS-PAGE) gels, and transferring them to nitrocellulose. During this procedure, RNA is partially hydrolyzed to a modal size of approximately 50-70 nucleotides, and at the end of the purification, protein is removed by proteinase $\mathrm{K}$, and RNA linkers are directionally added to the RNA, which is then PCR-amplified and sequenced. CLIP gives a snapshot of where Nova is bound in vivo; our initial studies identified binding sites present in large introns, binding sites in the vicinity of alternate exons, and binding sites in other regions (untranslated region, intergenic) and allowed us to validate seven new Nova splicing targets that could be validated in Nova KO brain (Ule et al. 2003). Our current analysis suggests that perhaps only about $10-20 \%$ of CLIP targets harbor high YCAY cluster score sequences, with the majority of the remaining RNAs harboring lower YCAY cluster scores (J. Ule and R.B. Darnell, unpubl.). This suggests that Nova may spend a considerable amount of its time sampling a wide range of RNAs for high-affinity binding sites; further studies will be required to assess whether Nova has any biologic role in the wider range of RNAs with weaker binding sites.

A second new approach to identifying Nova target RNAs arose through a collaboration with Affymetrix, which had developed a new alternative splicing microarray that was itself in need of validation. This microarray, a prototype for more comprehensive chips now under development, harbored 40,443 perfect match and mismatch probe sets spanning alternative exons; importantly, these included probe sets for both exon-included isoforms and the corresponding exon-skipped isoform. This proved to be essential, as statistical analysis of differences in exon inclusion or exclusion alone, comparing Nova KO and WT brain, only yielded a predictive power of about $20 \%$. As a result, we developed an algorithm, termed ASPIRE, in which we demanded reciprocal changes for any one putative Nova-regulated exon. By searching for such reciprocal changes in independent probe sets that measured exon inclusion and exon exclusion, our predictive power improved drastically. Using ASPIRE, we were able to validate 49 of 49 of our top predicted Nova-regulated exons identified in comparison of Nova KO and WT RNAs (Ule et al. 2005b).

The value of these two new methods is severalfold: First, the relatively large list of validated Nova targets provided a feed-forward data set to allow further target identification. Specifically, the ability to examine and compare known targets with control, alternatively spliced transcripts allowed the definition of the RNA map for genome-wide prediction of Nova activity. In addition, the larger set of validated targets has led us forward to be able to begin to look at Nova function with a comprehensive viewpoint.

\section{IMPLICATIONS DERIVED FROM A GLOBAL UNDERSTANDING OF RBP ACTION}

\section{Functional Considerations}

The beginning of a global understanding of the set of coregulated RNAs in the brain allows a new direction to be pursued: analysis of the functions encoded by those RNAs. From our early studies identifying GlyR $\alpha 2$ and $\mathrm{GABA}_{\mathrm{A}}$ transcripts as Nova targets and recognizing that they both encoded inhibitory neurotransmitter receptors, we have been aware of the possibility that Nova acts on a biologically restricted set of RNAs and that those RNAs might relate to the pathogenesis of the inhibitory motor dysfunction evident in patients with the paraneoplastic POMA syndrome. However, because these RNAs were not identified in an objective genome-wide screen, we 
were hesitant to make too much of these observations.

Nonetheless, we were further drawn to this observation after our first CLIP experiments. Analysis of 34 transcripts that had been identified multiple times in the 340 CLIP targets originally sequenced revealed that these too had a biologic coherence; $71 \%$ of these RNAs encoded proteins that function in the neuronal synapse (Ule et al. 2003). Since CLIP qualified as an unbiased, genomewide screen, this provided the first compelling evidence that Nova might regulate a restricted set of transcripts in the brain. Nonetheless, the sequencing of 340 CLIP tags cannot be considered an exhaustive analysis of Nova RNA targets. Although subsequent analysis of CLIP targets has continued to strengthen our original observations (J. Ule and R.B. Darnell, unpubl.), it was a genome-wide microarray analysis that provided overwhelming evidence regarding the nature of Nova RNA targets.

Our microarray screen for Nova-regulated alternative exons interrogated 40,443 exons in 7,175 transcripts; 49 Nova-regulated transcripts were identified and validated by RT-PCR. We analyzed the biologic functions encoded by these 49 transcripts using two approaches. In the first, we used the gene-ontology (GO) annotations of encoded functions, as a means of providing an unbiased assessment in a way capable of strict statistical analysis. This analysis revealed that Nova-regulated transcripts were highly enriched (in a statistically robust manner: $P$ $<0.001$, false discover rate 0.03 , with $6-13$-fold enrichment) in proteins that act at the cell junctions, suggesting that they were synaptic proteins (the most enriched GO categories were synapse biogenesis, synaptic transmission, cell-cell signaling, cortical actin organization beneath the membrane, cell adhesion, and extracellular matrix organization; Ule et al. 2005b).

These observations were further supported by individual annotation, searching PubMed for data regarding the biologic function of each validated Nova target. These data led to a confirmatory and more specific picture: The overwhelming majority (essentially all) of Nova targets encoded proteins functionally related to the neuronal synapse. No changes in steady-state levels were detected in the absence of Nova, indicating that Nova regulates the quality, but not the quantity, of a discrete set of synaptic proteins (Ule et al. 2005b).

This in turn suggests that Nova is likely to regulate the physiology of the synapse. Our first test of this hypothesis came from an evaluation of several Nova targets that had been repeatedly identified by CLIP, and whose encoded proteins were part of a new physiologic circuit being studied by Lily Jan and colleagues. This circuit represents a long-term inhibitory response to tetanic or coincidence (long-term potentiating [LTP]) stimuli. Jan and colleagues had found that LTP of slow inhibitory postsynaptic currents (sIPSC) were dependent on GIRK2, GABAB, and CaMKII proteins, all of which were encoded by transcripts that were Nova targets. LTP of sIPSC was therefore evaluated in Nova KO mice and was found to be completely and specifically absent (other parameters of synaptic function were normal; Huang et al. 2005). This study provided the first demonstration that identification of Nova RNA targets on a global scale can provide specific insights into physiologic function. Moreover, this study furthered the notion that RNA regulation has important roles in modulating synaptic plasticity (hippocampal LTP), which is thought to represent the physiologic correlate of complex information processing in the brain (Ule and Darnell 2006).

\section{Evolutionary Considerations}

A second set of issues arising from developing global insight into RBP function are evolutionary considerations. In the RNA world view, RNA molecules were the first informatic and enzymatic dual-function molecules to arise in evolution. How then did proteins evolve to harness the power of RNA? This question is directly approached in considering how RBPs evolved to regulate the complexity of information at a level that is specific to RNA molecules: alternative splicing.

Our studies with Nova point out some interesting issues in considering this problem. The Nova-binding site is rather low complexity-YCAY motifs occur on average every 64 nucleotides. This motif is even simpler than the characterized transcription-factor-binding sites, which are typically in the 6-8-nucleotide range. What is the relevance of such a finding?

Such low-complexity sequences are relatively easy to evolve by mutations. And the low constraints on such evolution - the only strict requirement in Nova KH binding is a CA dinucleotide - would thereby enable a larger fraction of the genome to sample the consequences of evolving a Nova-binding site, with those reaping a benefit able to increase, over time, the density of YCAY motifs. This in turn would solidify Nova binding, as its affinity for RNA targets increases with increasing density of YCAY motifs (Buckanovich and Darnell 1997; Yang et al. 1998; Jensen et al. 2000a; Dredge and Darnell 2003; Musunuru and Darnell 2004; Dredge et al. 2005). Thus, the number of Nova-regulated targets can grow as evolution generates greater complexity in the genome; for example, through duplication of exons or genes. In fact, preliminary analysis of the evolutionary conservation of Nova-regulated exons, suggesting a growing set of Nova-regulated exons through evolution from invertebrates to chick to mammals, is consistent with this idea (J. Ule et al., unpubl.).

One corollary to the idea that Nova-binding sequences may be widely dispersed and rapidly evolving is that Nova itself is tightly fixed in evolution. This fixation is essential, as mutations altering the recognition motif of Nova would simultaneously destroy the regulation of an array of crucial alternate exon information. In fact, the Nova KH domain is extremely tightly conserved down through invertebrates (Buckanovich et al. 1993; R.B. Darnell et al., unpubl.), suggesting that the ability to bind a YCAY motif became a powerful but unalterable facet of RBP-RNA regulation early in evolution. This turns RNA regulation on its head in a way that is in harmony with the idea of the RNA world: RNA remains the powerful emerging evolutionary force, whereas the protein regulators take on roles as inert drones to mediate the regulation that RNA demands. 
A second corollary is that a strict biologic coherence to transcripts harboring Nova-binding sites may be maintained and even refined through such a model. As we have observed, Nova targets are almost uncannily restricted to encoding proteins involved in synaptic biology. At the same time, it is clear that neurons (presumably through other factors) regulate alternative splicing of many other kinds of transcripts; in our analysis, the largest such groups, defined by the GO category, were transcripts encoding proteins involved in the regulation of metabolism, biosynthesis, and transcription, and yet Nova regulated no RNAs in these categories.

An interesting question for the future will be to explore the extent to which Nova might contribute to the complexity in the regulation of synaptic function between different neuronal types or even within a single neuron. It is clear that Nova-regulated exons respond to the presence of Nova in a dose-dependent manner and that different exons have a different threshold for Nova action. Thus, titration of Nova levels within a single neuron may lead to an array of actions on alternative exons, each tuned to a different degree of sensitivity to Nova levels. The correlation of Nova sensitivity with YCAY cluster scores may provide a means of evaluating this idea. Furthermore, there are many thousands of synapses within a single neuron, and the question arises as to whether Nova RNA regulation might be able to differentially modulate activity at one synapse relative to another. The exciting finding that Nova regulates LTP of sIPSC (Huang et al. 2005), together with the finding that Nova is present at neuronal synapses (R.B. Darnell et al., unpubl.), suggests that there may yet remain undiscovered dimensions to the ways in which RBPs may regulate the complexity of RNA expression.

\section{ACKNOWLEDGMENTS}

This work is an overview of the efforts of a large number of people and their experiments undertaken over the years. Although I have tried to cite the efforts of all, I would especially thank Steven Burley, Ron Buckanovich, Jennifer Darnell, Kate Dredge, Lily Jan, Kirk Jensen, Hal Lewis, Aldo Mele, Kiran Musunuru, Giovanni Stefani, and Jernej Ule for major contributions toward developing the key points in the development of the story told here. This work was supported by the National Institutes of Health (R01 NS34389 and NS40955 to R.B.D.) and the Howard Hughes Medical Institute. R.B.D. is an Investigator of the Howard Hughes Medical Institute.

\section{REFERENCES}

Albert M.L. and Darnell R.B. 2004. Paraneoplastic neurological degenerations: Keys to tumour immunity. Nat. Rev. Cancer 4: 36 .

Blencowe B.J. 2005. Splicing on the brain. Nat. Genet. 37: 796. . 2006. Alternative splicing: New insights from global analyses. Cell 126: 37.

Buckanovich R.J. and Darnell R.B. 1997. The neuronal RNA binding protein Nova-1 recognizes specific RNA targets in vitro and in vivo. Mol. Cell. Biol. 17: 3194.
Buckanovich R.J., Posner J.B., and Darnell R.B. 1993. Nova, the paraneoplastic Ri antigen, is homologous to an RNAbinding protein and is specifically expressed in the developing motor system. Neuron 11: 657.

Buckanovich R.J., Yang Y.Y., and Darnell R.B. 1996. The onconeural antigen Nova-1 is a neuron-specific RNA-binding protein, the activity of which is inhibited by paraneoplastic antibodies. J. Neurosci. 16: 1114.

Darnell R.B. 1996. Onconeural antigens and the paraneoplastic neurologic disorders: At the intersection of cancer, immunity and the brain. Proc. Natl. Acad. Sci. 93: 4529.

2002. RNA logic in time and space. Cell 110: 545.

Darnell R.B. and Posner J.B. 2003a. Observing the invisible: Successful tumor immunity in humans. Nat. Immunol. 4: 201.

2003b. Paraneoplastic syndromes involving the nervous system. N. Engl. J. Med. 349: 1543.

. 2006. Paraneoplastic syndromes affecting the nervous system. Semin. Oncol. 33: 270.

Dredge B.K. and Darnell R.B. 2003. Nova regulates GABA(A) receptor gamma2 alternative splicing via a distal downstream UCAU-rich intronic splicing enhancer. Mol. Cell. Biol. 23: 4687.

Dredge B.K., Stefani G., Engelhard C.C., and Darnell R.B. 2005. Nova autoregulation reveals dual functions in neuronal splicing. EMBO J. 24: 1608 .

Green R., Ellington A.D., Bartel D.P., and Szostak J.W. 1991. In vitro genetic analysis: Selection and amplification of rare functional nucleic acids. Methods 2: 75.

Huang C.S., Shi S.H., Ule J., Ruggiu M., Barker L.A., Darnell R.B., Jan Y.N., and Jan L.Y. 2005. Common molecular pathways mediate long-term potentiation of synaptic excitation and slow synaptic inhibition. Cell 123: 105.

Jensen K.B., Musunuru K., Lewis H.A., Burley S.K., and Darnell R.B. 2000a. The tetranucleotide UCAY directs the specific recognition of RNA by the Nova KH3 domain. Proc. Natl. Acad. Sci. 97: 5740.

Jensen K.B., Dredge B.K., Stefani G., Zhong R., Buckanovich R.J., Okano H.J., Yang Y.Y., and Darnell R.B. 2000b. Nova1 regulates neuron-specific alternative splicing and is essential for neuronal viability. Neuron 25: 359 .

Lewis H.A., Musunuru K., Jensen K.B., Edo C., Chen H., Darnell R.B., and Burley S.K. 2000. Sequence-specific RNA binding by a Nova KH domain: Implications for paraneoplastic disease and the fragile X syndrome. Cell 100: 323.

Lewis H.A., Chen H., Edo C., Buckanovich R.J., Yang Y.Y., Musunuru K., Zhong R., Darnell R.B., and Burley S.K. 1999. Crystal structures of Nova-1 and Nova-2 K-homology RNAbinding domains. Structure 7: 191.

Mili S. and Steitz J.A. 2004. Evidence for reassociation of RNAbinding proteins after cell lysis: Implications for the interpretation of immunoprecipitation analyses. RNA 10: 1692.

Musunuru K. and Darnell R.B. 2001. Paraneoplastic neurologic disease antigens-RNA-binding proteins and signaling proteins in neuronal degeneration. Annu. Rev. Neurosci. 24: 239. . 2004. Determination and augmentation of RNA sequence specificity of the Nova K-homology domains. Nucleic Acids Res. 32: 4852.

Newman L.S., McKeever M.O., Okano H.J., and Darnell R.B. 1995. $\beta$-NAP, a cerebellar degeneration antigen, is a neuronspecific vesicle coat protein. Cell 82: 773 .

Rosenfeld M.G., Mermod J.J., Amara S.G., Swanson L.W., Sawchenko P.E., Rivier J., Vale W.W., and Evans R.M. 1983. Production of a novel neuropeptide encoded by the calcitonin gene via tissue-specific RNA processing. Nature 304: 129.

Stamm S., Zhang M.Q., Marr T.G., and Helfman D.M. 1994. A sequence compilation and comparison of exons that are alternatively spliced in neurons. Nucleic Acids Res. 9: 1515.

Szabo A., Dalmau J., Manley G., Rosenfeld M., Wong E., Henson J., Posner J.B., and Furneaux H.M. 1991. HuD, a paraneoplastic encephalomyelitis antigen contains RNAbinding domains and is homologous to Elav and sex lethal. Cell 67: 325 .

Szostak J.W. and Ellington A.D. 1993. In vitro selection of func- 
tional RNA sequences. In The RNA world (ed. R.F. Gesteland and J.F. Atkins), p. 511. Cold Spring Harbor Laboratory Press, Cold Spring Harbor, New York.

Ule J. and Darnell R.B. 2006. RNA binding proteins and the regulation of neuronal synaptic plasticity. Curr. Opin. Neurobiol. 16: 102.

Ule J., Jensen K., Mele A., and Darnell R.B. 2005a. CLIP: A method for identifying protein-RNA interaction sites in living cells. Methods 37: 376.

Ule J., Jensen K.B., Ruggiu M., Mele A., Ule A., and Darnell R.B. 2003. CLIP identifies Nova-regulated RNA networks in the brain. Science 302: 1212.
Ule J., Stefani G., Mele A., Ruggiu M., Wang X., Taneri B., Gaasterland T., Blencowe B.J., and Darnell R.B. 2006. An RNA map predicting Nova-dependent splicing regulation. Nature (in press).

Ule J., Ule A., Spencer J., Williams A., Hu J.S., Cline M., Wang H., Clark T., Fraser C., Ruggiu M., et al. 2005b. Nova regulates brain-specific splicing to shape the synapse. Nat. Genet. 37: 844.

Yang Y.Y.L., Yin G.L., and Darnell R.B. 1998. The neuronal RNA binding protein Nova-2 is implicated as the autoantigen targeted in POMA patients with dementia. Proc. Natl. Acad. Sci. 95: 13254 . 


\title{
$\$_{\text {SSH }}^{\infty}$ \& Cold Spring Harbor Symposia SYMPOSIA
}

\section{Developing Global Insight into RNA Regulation}

\author{
R.B. DARNELL
}

Cold Spring Harb Symp Quant Biol 2006 71: 321-327

Access the most recent version at doi:10.1101/sqb.2006.71.002

$\begin{array}{ll}\text { References } & \begin{array}{l}\text { This article cites } 31 \text { articles, } 9 \text { of which can be accessed free at: } \\ \text { http://symposium.cshlp.org/content/71/321.full.html\#ref-list-1 }\end{array}\end{array}$

\section{License}

Email Alerting Receive free email alerts when new articles cite this article - sign up in Service the box at the top right corner of the article or click here. 